La educación secundaria, en el marco de la política del Estado de Hidalgo. Secondary education, within the framework of the policy of the State of Hidalgo.

Margarita Zamora Hernández

Universidad la Salle Pachuca

Nota sobre el autor

Maestra en administración educativa. Profesora frente a grupo en secundaria.

Esta investigación fue financiada con recursos del autor.

Recibido:1/1/2006

Aceptado:1/1/2006

Derechos de autor (C) 2006 Universidad La Salle Pachuca - Todos los derechos reservados.

Condiciones de Uso: La reproducción total o parcial de la obra en soporte electrónico queda prohibida sin la autorización de la Universidad la Salle Pachuca. 


\title{
La educación secundaria, en el marco de la política del Estado de Hidalgo.
}

\author{
Margarita Zamora Hernández *
}

\begin{abstract}
Resumen
La pretensión del presente artículo es reflexionar acerca de la situación que guarda la educación secundaria en nuestra entidad; así como describir algunas de sus implicaciones políticas, económicas y sociales en el ámbito hidalguense. Para dar cuenta de cómo se han llevado a la realidad las utopías educativas en las últimas cuatro décadas.

La metodología de indagación en la construcción del diagnóstico, tiene su fundamento en un trabajo etnográfico, con base en entrevistas, observación libre, consulta a informantes clave y la revisión de documentos necesarios. En la valoración académica de alumnos, se consideró al INEE 2002-2003, en docentes al ProNap 1997-2004. Para el estudio y valoración de datos, es retomado el análisis estratégico; en este caso pretende una valoración crítica, que permita la toma de decisiones a partir de la técnica FODA. En las conclusiones son recuperados elementos significativos que pretenden orientar el reposicionamiento de las secundarias hidalguenses.
\end{abstract}

\begin{abstract}
The pretension of the present article is to reflect about the situation that keeps the secondary education in our organization; as well as to describe some of its political, economic and social implications in the hidalguense scope. In order to give account of how the educative utopias in last the four decades have taken to the reality. The methodology of investigation in the construction of the diagnosis, has its foundation in an ethnographic work, with base in interviews, free observation, it consults informants nails and the necessary document revision. In the academic valuation of students, it was considered to INEE 2002-2003, in educational to ProNap 1997-2004. For the study and valuation of data, the strategic analysis is retaken; in this case it tries a critical valuation, that allows the decision making from technique FODA. In the conclusions significant elements are recovered that they try to orient the repositioning of the secondary hidalguenses. Key words: Secondary education, educational, students, policy, science.
\end{abstract}

\section{Introducción}

La educación en el Estado de Hidalgo, al igual que en el resto de la Republica Mexicana, manifiesta problemáticas similares en sus tres niveles. El 
presente artículo centra su atención en las contingencias que se dan al interior del primer nivel que es educación básica, donde se ubica la secundaria en tres modalidades: generales, técnicas y telesecundarias. El análisis se llevó a cabo durante el semestre de julio a diciembre de 2005.

Se consideró de los resultados emitidos por el INEE, (Instituto Nacional de Evaluación Educativa) sobre pruebas nacionales y estatales de aprovechamiento en lectura y matemáticas 2002-2003, lo referente a los estudiantes hidalguenses. La información sobre saberes y capacitación docente fue obtenida del Programa estatal ProNAP (Programa Nacional de Actualización Profesional) 1997-2004, lo cual posibilitó un mayor acercamiento sobre los niveles de conocimiento de alumnos, docentes y directivos.

La recuperación de información hizo posible la construcción del diagnóstico a secundarias mediante la técnica FODA (Fortalezas, Oportunidades, Debilidades y Amenazas), que se complementó con entrevistas, registros de observación directa a docentes, directivos y alumnos.

Las acciones emprendidas fueron guiadas mediante Planeación estratégica y desde la óptica de Steiner quien señala que una de las condiciones fundamentales para la Planeación estratégica: "es sistemática en el sentido de que es organizada y conducida con base en una realidad entendida" (Steiner 2002: 21).

Lo cual nos lleva a establecer prioridades en el estudio, a fin de dar cuenta fehaciente de la situación en torno a un diagnóstico, para la mejor toma de decisiones.

El plan de acción, indica previsión ¿qué pude hacerse?, planeación ¿qué se va a hacer?, dirección ¿cómo ve que se haga?, control ¿cómo se está realizando?. Lo cual permitió guiar el proceso desde el diagnóstico hasta las conclusiones.

\section{Desarrollo}


Para fundamentar este trabajo, se toman como marco referencial a dos autores que actualmente desarrollan investigación educativa en torno a secundarias en nuestro país Rafael Quiroz Estrada y Etelvina Sandoval Flores. Esta última se retoma en Medina (2000: 264), al afirmar que "la secundaria ha sido un espacio poco investigado en nuestro país y esa falta de conocimiento repercute en la eficacia de muchas de las propuestas que impulsan las nuevas políticas educativas, pues suelen estar ajenas a las especificidades de este nivel". Sólo así, se encuentra la justificación de múltiples programas gubernamentales de apoyo educativo en la entidad; sin considerar las necesidades específicas de la secundaria, porque es tratada en la mayoría de estudios como una institución más del nivel educativo (preescolar y primaria).

Este estudio hace referencia específica a la secundaria, porque es notoria la baja calidad de la enseñanza, la deserción escolar, la baja eficiencia terminal, para lo cual desde esta perspectiva se plantea:

¿Cuáles son las expectativas de la secundaria en la sociedad mexicana? según Quiroz (2000), la secundaria tiene como debe ser, el ideal de equidad y pertinencia que proporcione aprendizajes significativos, funcionales, duraderos $y$ equivalentes para todos los jóvenes del país de 15 ó 16 años de edad. Pero, el mismo autor después de una minuciosa investigación, señala que dicho ideal está lejos de ser realidad, ya que de 100 niños que ingresan a primaria 74 la terminan, de estos 74 se inscriben 65 en secundaria y sólo 61 la concluyen en 3 años. El panorama es desalentador, e indica la necesidad de hacer revisiones profundas, sobre todo si consideramos que en la actualidad la eficiencia terminal en nuestra entidad es baja. Lo que implica una mayor atención por parte de las autoridades educativas y civiles, en relación a las causas que provocan estos resultados adversos, además de interesarse en las problemáticas que atañen a la secundaria, y que pueden estar relacionadas desde el Plan y programa de estudio 
SEP (1993), en relación con el currículo, por anacrónico y denso en contenidos; hasta la participación política de los actores del proceso educativo.

Por todo lo anterior, es importante no perder de vista que la secundaria "es un espacio indefinido entre la educación primaria y el bachillerato, un puente que posterga la inserción en el mundo laboral, pero que tampoco capacita adecuadamente para continuar el trayecto hacia una escolaridad superior" Sandoval citada en Medina (2000: 264). Esto se trae a colación porque en nuestra entidad, de acuerdo a las políticas educativas de cada sexenio se visualiza un tipo de mexicano, que responda a las necesidades de la estructura económica vigente. Esto es, la producción de mano de obra barata destinada al sector terciario, realidad que es palpable porque una de las posibilidades mayoritarias entre los jóvenes, es cruzar la frontera durante o poco después de la secundaria, por diversas problemáticas. Entre las que podemos enumerar reprobación de asignaturas, imposibilidad de poder acceder al siguiente nivel educativo, al no acreditar el examen de ingreso, la ausencia de fuentes de empleo en su localidad, que puede relacionarse con la implementación de un sistema educativo cuestionable en su operatividad, al considerar los requerimientos y condiciones sociales actuales del país.

Es necesario enfatizar que una sociedad altamente educada será capaz de una transmisión cultural con la visión humanística de acuerdo a Bourdieu y Passeron (1967). Lo que implicaría que los jóvenes alcanzaran mayor grado de escolaridad; por los altos índices del conocimiento que se juegan en el contexto internacional. En donde la exclusión y discriminación por falta de habilidades cognitivas, conduce a la marginación de pueblos, estados y países, al imperar modelos educativos de países desarrollados, con una cultura de globalización como eje rector.

\section{Diagnóstico situacional}


El incursionar en la educación básica, a través de la secundaria pretende mostrar que el desarrollo educativo no sólo depende de las condiciones propias de cada modalidad sino que, los efectos del entorno, también son determinantes en los procesos y sus tendencias, lo cual se manifiesta en la situación de nuestra entidad y sus implicaciones en el ámbito nacional. Al respecto, el Ceneval (2005), aporta un estudio donde plantea a México dividido en tres regiones; la primera agrupa al $40 \%$ de la población en zonas con un nivel bajo de conocimientos, entre las que se encuentra el Estado de Hidalgo. Un 20\% en zonas de nivel medio de conocimiento, y el $40 \%$ restante en zonas con un nivel alto de conocimiento. El mismo estudio advierte que Hidalgo, Puebla, San Luís Potosí, Yucatán y Zacatecas registran niveles bajos de conocimientos. Pero, pueden aumentarlo de acuerdo a sus características generales. Esta información deberá alentar la posibilidad de que los actores políticos apoyados en la investigación educativa propongan nuevas estrategias, para la movilidad del conocimiento que permita un desarrollo equitativo, con una visión prospectiva de producción; sobre viejas prácticas paternalistas.

En el Estado de Hidalgo, al igual que en el resto del país escasean estudios sobre educación secundaria, sin embargo, se han considerado en este trabajo algunos datos, sobre el contexto que muestran aspectos del panorama estatal, para un mayor acercamiento a la realidad educativa de secundarias. En Hidalgo el censo del año 2000, presentó un universo poblacional de 1, 973968 de las cuales 339 866 hablan lengua indígena y representan $17.21 \%$. Sobresalen los municipios de Xochiatipan, Jaltocán, Yahualica, Huautla, Atlapexco, y Huazalingo, al menos tres cuartas partes de sus habitantes hablan lengua indígena, en todos los municipios de la entidad hay al menos una persona que se comunica en lengua indígena, IHE (2005). Es decir, nuestro Estado muestra un mosaico cultural, que involucra a su población joven, que desafortunadamente después de la primaria rompe la 
continuidad de atención a la diversidad cultural, con escasa o nula atención a los jóvenes de regiones menos favorecidas.

Se puede inferir que la atención educativa en el Estado de Hidalgo durante las últimas cuatro décadas ha atendido la cobertura en detrimento de la calidad, por lo que, se hace necesaria una mayor atención a la calidad educativa por parte de todos los sectores sociales, lo que abre la interrogante:

¿cuál ha sido la participación de organizaciones que influyen directamente en los impactos educativos?, inicialmente se puede reconocer que la instancia sindical del magisterio, mantiene una subordinación a decisiones y acciones de partidos o “líderes" políticos Touraine (1989: 268), enfrascados en sus propios intereses por dominar cotos de poder, lo que implica que sindicato, gobierno y partidos políticos se interesen poco, o nada en la formación de una juventud que aspira a mejores posibilidades desde las aulas de las secundarias hidalguenses.

\section{Valoración de Secundarias en Hidalgo}

La descripción del estado actual de las secundarias, mediante FODA, posibilita una valoración prospectiva Miklos y Tello (1993), desde la variable interna controlable en fortalezas y debilidades; y externa no controlable, pero si prevenible con oportunidades y amenazas Romero (2001: 9). En algunos hallazgos se menciona brevemente el origen de la información, que hizo posible la construcción del diagnóstico situacional de secundarias, soslayando la subjetividad que subyace a la interacción humana.

\section{Fortalezas}

Este primer apartado, es considerado como una variable controlable desde el ámbito interno, porque se visualizan los argumentos plausibles de la organización. Además, es ponderable su análisis desde la óptica de costeproducto. Al respecto se enumeran las siguientes fortalezas. 
$>$ Los alumnos que ingresan a la secundaria, ya poseen conocimientos previos en la mayoría de las asignaturas. Entrevistas y documentos.

> Financiamiento a programas educativos, desde distintos ámbitos, estatal, federal e internacional. Revisión de documentos.

> Construcción y rehabilitación de espacios educativos. Observaciones

> Equipamiento de aulas con mobiliario y material didáctico.

> En algunos casos los salarios y prestaciones de docentes, son mejores, comparados en percepciones de educación media. Entrevistas a docentes.

> En ciertas modalidades de secundaria se cuenta con varias categorías en puestos directivos. Revisión de documentos.

> La comunidad educativa integrada por alumnos, docentes, directivos y apoyos a la educación, se caracteriza por tener apego a la norma institucional. Observación directa en reuniones de personal.

$>$ El potencial físico, intelectual y cultural de los jóvenes. Entrevistas en escuelas.

> Las secundarias en sus tres modalidades, participan en procesos de evaluación académica con distintos resultados. Informes del INNE. Se deduce, que en educación secundaria se cuenta con programas y recursos económicos, pero se hace necesario analizar la eficiencia y eficacia en resultados con base en coste-beneficio, a fin de reorientar las deficiencias y plantear nuevas estrategias.

\section{Debilidades}

Para la planeación estratégica, es fundamental descubrir cuales son las debilidades de un proyecto a fin de establecer mecanismos que subsanen durante el proceso, un mejor desarrollo del proyecto, para tal efecto desde el interior, se considera una variable controlable. En el caso que nos ocupa, se pueden enunciar las siguientes debilidades. 
> Una creciente "simulación" sobre el proceso educativo, sustentada en la burocracia actual. Observación libre.

> La capacitación docente no cubre las necesidades de profesionalización, por atender intereses meritocráticos de acuerdo a datos del ProNAP estatal.

> La disfunción existente entre los conocimientos sobre tecnología de punta, y los conocimientos tradicionales del docente, no responden a las expectativas de los alumnos. Observación libre.

> Escaso compromiso profesional y ético, en gran parte de los sujetos relacionados con la toma de decisiones en el ámbito político y educativo de acuerdo al rol asignado. Entrevistas.

> Docentes sin dominio pleno de contenidos académicos apoyados en un creciente enciclopedismo, donde el libro de texto, juega el roll principal al interior del aula, con base en observaciones directas a profesores frente a grupo.

$>$ En resultados del ProNAP 1997-2004, se detecta que en el Estado de Hidalgo los directivos escasamente reconocen la currícula, para orientar académicamente a los docentes a su cargo. Inmersos en la "trama" de la función administrativa como lo enuncia Sandoval (2001). Lo cual repercute al interior del aula.

$>$ Asignación de presupuesto económico a programas educativos, sin estudios rigurosos de factibilidad ni seguimiento. Documentos y entrevistas a directivos.

> La capacitación no logra la profesionalización de docentes y directivos, de acuerdo a la función desempeñada. Resultados del ProNAP y entrevistas realizadas.

> Escasa eficiencia de recursos humanos en áreas académicas y administrativas en alusión al coste beneficio. Observación libre. 
> Minimizar la calidad de la educación secundaria, al incrementar telesecundarias. Hidalgo uno de los Estados con mayor porcentaje de esta modalidad en el país. Revisión de documentos.

> Desde el ámbito político y gubernamental se fomenta la estatización de la movilidad social en beneficio de intereses minoritarios. Asignación de puestos directivos por compromisos políticos. Observación libre.

> La fragmentación y extensión de contenidos curriculares dificultan la apropiación de los mismos. Entrevistas a jóvenes de secundaria.

> Un creciente aislamiento de docentes, como resultad de la "especialización" por asignatura en secundaria. Observación, entrevista y revisión de documentos.

> Los resultados académicos en secundarias técnicas, por debajo de la media nacional, telesecundarias registra los índices más bajos de las tres modalidades. Resultados del INEE 2002-2003.

\section{Amenazas}

Las amenazas en todo el proceso de un proyecto están latentes y se relacionan a la cuestión volitiva de los participantes, con las organizaciones sociales que se generan en las interrelaciones de operatividad, las condiciones laborales, la logística de los objetivos. Desde la perspectiva externa se consideran no controlables, pero si previsibles que ayudan a la toma de decisiones institucionales y pueden enumerarse las siguientes.

> Continuar con programas de apoyo, sin considerar realmente necesidades de alumnos, docentes, instituciones y contexto hidalguense. Observaciones y análisis de discurso.

> Formación en secundaria de jóvenes, destinados al sector terciario de acuerdo al informe del INEE 2002-2003, por los resultados académicos. 
> Desarraigar desde la base educativa la ciencia y tecnología, por la creciente "matematifobia", informe del INEE 2002-2003.

> Apropiarse individual y colectivamente de un discurso triunfalista que manifiesta "estamos bien", sin reconocer las grandes deficiencias educativas en el Estado de Hidalgo. Análisis de discursos.

> A corto, mediano y largo plazo escasas posibilidades de desarrollo y movilidad social, por la deficiente atención e indiferencia de actores políticos en torno al hecho educativo de educación básica. Observación libre.

> Continuar con el fenómeno de emigración en gran parte de la población, pero con mayor incidencia y preocupación en adolescentes. Documentos y entrevistas.

> Desconocimiento de la multiculturalidad estatal, en alusión al currículo único. Entrevista, observación y revisión de documentos.

\section{Oportunidades}

Es muy importante, para la planeación y organización de una empresa, el conocimiento de las oportunidades que se movilizan desde el exterior, en el que se interactúa. Las oportunidades al igual que amenazas, son una variable no controlable, si prevenible, al modificar juicios en la toma de decisiones. Así, en las secundarias hidalguenses se pueden considerar las siguientes.

> La disposición de la comunidad educativa, en la dinámica del deber ser y de los compromisos individual y colectivo, posibilita la transformación cualitativa de los procesos educativos, que determine el reposicionamiento de la educación secundaria. Observación libre.

> Impulsar las bases del conocimiento científico, desde la educación básica. Explorar la beta de la investigación educativa en secundaria como un 
campo de múltiples posibilidades, para el desarrollo de los otros niveles educativos.

> Propiciar la posibilidad del acercamiento al desarrollo tecnológico, a partir de la educación básica.

> Dignificar la función sindical, con una perspectiva ideológica, acorde a las verdaderas necesidades educativas de una sociedad posmoderna.

> Que el Estado y los grupos políticos, consideren a la secundaria como una vía de desarrollo científico y tecnológico, a corto, mediano y largo plazo.

\section{Conclusiones}

A partir de FODA se puede inferir que la situación educativa, de las secundarias en el Estado de Hidalgo, enfrenta múltiples retos, que atañen a distintos actores sociales. Los cuales deberán intervenir decididamente, con creatividad, compromiso e imaginación.

A través del estudio, se reconoce que la perspectiva interna permea en mayor grado, más aún en las debilidades, sin olvidar que en la planeación estratégica son consideradas variables controlables. En relación al factor externo las amenazas causan la desestabilización de las secundarias, que en este caso superan a las oportunidades, consideradas como las que posibilitan el desarrollo del proceso. Los ámbitos de FODA por su parte, responden a una temporalidad, esto es, las fortalezas no son para siempre y las oportunidades, no son permanentes.

De manera general, el trabajo posibilita el planteamiento de ciertas sugerencias orientadas a la toma de decisiones como; subsanar los vicios de tradición sindical, que lesionan y minimizan los propósitos educativos, en detrimento de la educación básica y la sociedad hidalguense. 
Reconocimiento en forma y fondo de la interculturalidad juvenil, con un trato responsable, incluyente y respetuoso, hacia la búsqueda de estrategias acordes a la modernidad competitiva en ciencia y tecnología.

Implementar análisis prospectivos sobre la realidad hidalguense, sus

problemáticas, necesidades y aciertos; desde distintos ámbitos y contextos, para evitar así, la creación de dos Hidalgos: uno de marginación y otro en desarrollo. Al interior de las instituciones educativas, la tarea es amplia, porque es ahí, donde se deberán generar las transformaciones, basadas en sus recursos humanos. Desde el ámbito político y gubernamental reconocer los fines axiológicos y teleológicos de la educación básica, vinculados con las necesidades contextuales hidalguenses, para definir las interrogantes ¿qué?, ¿por qué?, ¿para qué?, ¿cuándo? y ¿dónde? de la educación secundaria.

Finalmente, es preciso pugnar por una cultura de evaluación, en todos las áreas educativas como uno de los medios, para lograr la eficiencia y eficacia educativa hidalguense.

Referencias

SANDOVAL, Etelvina. (2001), La trama de la escuela secundaria: institución, relaciones y saberes. Plaza y Valdés. México.

QUIROZ, Rafael. (2000), Las condiciones de posibilidad de aprendizaje de los adolescentes en la educación secundaria, tesis doctoral CINVESTAV-UPN México.

BOURDIEU, P., y PASSERON, J. C. (1967), La reproducción. Editorial Popular. Barcelona.

MEDINA, Carrasco Gabriel. (2000), Aproximaciones a la diversidad juvenil. COLMEX. México.

TOURAINE, Alain. (1989), América Latina Política y sociedad. Espasa-Calpe. Madrid.

STEINER, A. G. (2002), Planeación Estratégica. CECSA. México. MIKLOS, T. y TELLO M. (1993), Planeación prospectiva: una estrategia para el diseño del futuro. Limusa-Noriega. México. 
ROMERO, Flores J. Jesús. (2001), La evaluación institucional a través del análisis estratégico. SEP-Michoacán.

SEP. (1993) Plan y programas de estudio. México.

INEE. (2005), La inteligencia colectiva de México.

INEE. (2004), Resultados educativos: La secundaria 2002-2003.

IHE. (2005) Propuesta: Creación del instituto estatal de lenguas indígenas. 